\title{
Spectrophotometry of Trans-Neptunian Objects
}

\author{
Jennifer Romon, Alain Doressoundiram, and Maria Antonietta Barucci \\ DESPA, Observatoire de Meudon, 5 place Janssen, 92195 Meudon \\ Principal Cedex, France. E-mail: jennifer.romon@obspm.fr
}

\begin{abstract}
The Trans-Neptunian Objects (TNOs) are very difficult to observe due to their faintness. Spectra can be obtained for only few objects. For this reason, broad band photometry is the only available technique to study their physical properties from a statistical point of view. To improve the knowledge about the physical nature of the Kuiper Belt, we have obtained several observing runs at different four-meter class telescopes (NTT - Chile; CFHT - Hawaii).

The data presented here were obtained at NTT (ESO - Chile) during three observing runs from 1997 to 2000 . We used the SUSI and SUSI 2 CCD cameras with the Bessel B, V, R, and I filters.
\end{abstract}

\section{Data reduction method}

As the observed objects have visual magnitudes that range from 21 to 24 , data reduction must be performed very carefully. For this reason, we studied the various error sources on magnitude measurements and we compared the existing reduction techniques. The classical method consists in using an aperture large enough to include the majority of the flux of the object, as well as a significant contribution from the sky background. For objects as faint as TNOs, the error from the sky background dominates. The alternative method we have considered, called aperture correction method, consists in measuring the flux of the object with a smaller aperture, in order to limit the sky background contribution. As we use a small aperture, only a fraction of the flux is included, and it is required to perform an aperture correction. The correction to apply is computed using several bright stars in the same frame as the object. We compared both methods in order to choose the most appropriate one. We concluded that, due to the error on the correcting factor (which does not depend on the object brightness), the best method depends on the magnitude of the source: for bright objects $(V<20$ for these observations), the classical method should be used, whereas faint objects $(V>20)$ require the aperture correction (Barucci et al. 2000).

\section{Results}

We present here the results obtained for 18 TNOs. We have obtained an homogeneous set of optical broadband colors tor these objects. Figure 1 gives their $B-V$ vs. $V-R$ and $V-R$ vs. $R-I$ color distribution. The observed ob- 
jects present a wide spread of colors ranging from nearly neutral to very red. Our results do not confirm the bimodal color distribution previously found by Tegler \& Romanishin (1998). The difference in colors increases in the IR range, and diversity in near-IR colors is more striking than in optical colors. For this reason, observations in the near-IR range are discriminant to study the color distribution.
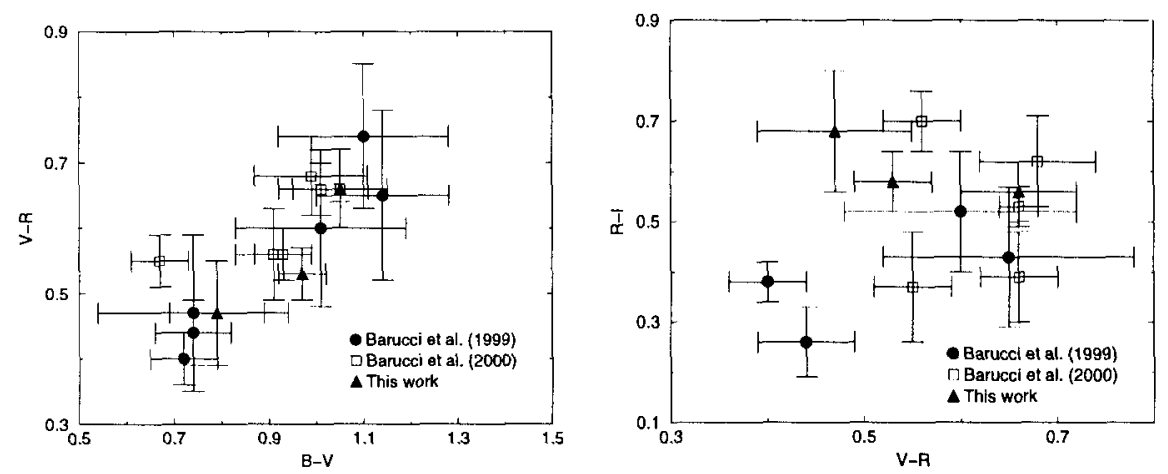

Figure 1. Color plots of TNOs: $B-V$ vs. $V-R$ and $V-R$ vs. $R-I$.

Our results confirm the color diversity previously suggested. The differences in colors may not be genetic, but are more probably due to different evolutions of these objects (impact resurfacing, cometary activity). The next step of these photometric studies is to derive a taxonomy in the same way as performed for the main belt asteroids. This requires a data sample extended to the near-IR colors as they are determinant variables for the statistical analysis.

\section{References}

Barucci, M. A., Doressoundiram, A., Tholen, D. J., Fulchignoni, M., \& Lazzarin, M. 1999, Icarus, 142, 476

Barucci, M. A., Romon, J., Doressoundiram, A., \& Tholen, D. J. 2000, AJ, 120, 496

Tegler, S. C., \& Romanishin, W. 1998, Nature, 392, 49 